\title{
The Management of Sciatica by Acupuncture: An Expert Consensus Using the Improved Delphi Survey
}

\author{
$\mathrm{Na}$ Zhang ${ }^{1,2}$ \\ Li-Qiong Wang' \\ Jin-Ling Li' \\ Xin-Tong Su (D) \\ Fang-Ting $\mathrm{Yu}^{\prime}$ \\ Guang-Xia Shi ${ }^{1}$ \\ Jing-Wen Yang' \\ Cun-Zhi Liu'
}

'International Acupuncture and Moxibustion Innovation Institute, School of Acupuncture-Moxibustion and Tuina, Beijing University of Chinese Medicine, Beijing, People's Republic of China;

${ }^{2}$ School of Acupuncture-Moxibustion and Tuina, Shandong University of Chinese Medicine, Shandong, People's Republic of China
Correspondence: Cun-Zhi Liu School of Acupuncture-Moxibustion and Tuina, Beijing University of Chinese Medicine, II Bei San Huan Dong Lu, Chaoyang District, Beijing 100029, People's Republic of China Email Icz_tg@I26.com
This article was published in the following Dove Press journal: Journal of Pain Research

Objective: Acupuncture therapy is an effective non-drug therapy for sciatica, but it has not yet formed an effective treatment strategy and recommendations. Our objective was to establish an expert consensus on acupuncture treatment of sciatica for clinical guidance based on the improved Delphi survey.

Methods: A group of 80 clinical specialists was invited to participate in two rounds of semiopen clinical issue investigation. At the same time, the PubMed, Embase, and Cochrane Library databases were searched for systematic reviews on acupuncture treatment of sciatica, and the quality of evidence was evaluated. Then the three-round Delphi survey was undertaken with 30 experts based on the clinical issue investigation and systematic reviews.

Results: In round 1 of the Delphi survey, the experts evaluated 17 items identified from the results of the clinical investigation and literature review. The criterion for achieving consensus was a threshold of $80 \%$ agreement. After the three-round Delphi survey, 16 items $(94.12 \%)$ achieved consensus, including 5 domains: the principle of acupuncture treatment for sciatica; the "dose" of acupuncture; the clinical effects of acupuncture; the adverse effects, and others. Conclusion: This Delphi survey achieved an expert consensus on key items in the management of sciatica for acupuncture, which provides the current opinions in China. We trust that these treatment recommendations may facilitate their implementation in the future.

Keywords: acupuncture, sciatica, expert consensus, Delphi survey

\section{Introduction}

Sciatica is usually unilateral radiation pain along the direction of the sciatic nerve, ${ }^{1,2}$ accounting for $13 \%$ to $40 \%$ in different studies and reviews. ${ }^{3}$ Lumbosacral nerve root (L4-S1) inflammation or compression is the main cause. ${ }^{4,5}$ Neuroradiology studies have confirmed that $85 \%$ of sciatica is related to disc disease. ${ }^{6}$ Most patients report low back pain at the same time as pain. The severity of pain varies from patient to patient along with a sudden or chronic onset mostly. ${ }^{2}$ It can extend from below the knee to the toes of the feet. ${ }^{7}$ In severe cases, symptoms such as low back pain, decreased muscle strength, and abnormal reflexes may occur, which would cause severe discomfort with limited quality of life and function. ${ }^{8}$

The current focus of treatment is to prevent the disease from becoming chronic through conventional drug treatment and early conservative treatment. ${ }^{9,10}$ However, conventional drugs have a variety of potential adverse events, particularly gastrointestinal and cardiovascular effects. ${ }^{11,12}$ Recently updated clinical guidelines emphasize the 
role of conservative treatment of sciatica from Denmark, and the US. ${ }^{13,14}$ Surgery which can early relieve pain maybe is an option if the symptoms do not improve after 6-8 weeks of early intervention, but its effect is similar to that of conservative treatment within one year. ${ }^{7,15}$

Acupuncture is a non-pharmacological therapy used in pain control. At present, acupuncture is widely used in the treatment of sciatica, but the National Institute for Health and Clinical Excellence (NICE) guidelines recommend that acupuncture should not be provided as a non-invasive treatment for patients with sciatica, which is based on the following: i) Relative values of different outcomes; ii) Trade-off between clinical benefits and harms; iii) Trade-off between net clinical effects and costs; iv) Quality of evidence; and v) Other considerations. ${ }^{16}$ In terms of the standardized use of acupuncture, there is no internationally recognized evidence and guidance recommendations except for the "Evidence-Based Acupuncture Clinical Practice Guide: Sciatica" from China. ${ }^{17}$ However, with the continuous emergence of new research evidence (ie, changes in available interventions, clinical efficacy, or cost), it is necessary to conduct expert consensus based on existing evidence and update appropriately to maintain the trustworthiness of clinical guidelines.

These uncertainties of acupuncture treatment recommendations for sciatica prompted this study to establish an expert consensus based on the clinical investigation and literature review by using the improved Delphi method, which is conducive to unifying the acupuncture principles and putting forward a standard therapeutic protocol for the treatment of sciatica which could be used in future trials and clinical practice.

\section{Methods}

This study was composed of four phases: (1) assistant team selection, (2) the clinical issue investigation, (3) literature review provided evidence support, (4) Delphi survey. The list development process is shown in Figure 1.

\section{The Selection of Assistant Team}

Our assistance team was composed of two methodological experts (WC and L-Q W), three acupuncture experts (C-Z L, HH, and YL), and three acupuncture Ph.D. (NZ, $\mathrm{J}-\mathrm{L} \mathrm{L}$, and $\mathrm{X}-\mathrm{T} \mathrm{S}$ ). The main responsibility of them was to set up the clinical questionnaires and consensus voting lists, and the statistical analysis of Delphi survey results.

\section{The Clinical Issue Investigation}

The Generation of the Initial Clinical Issues

After reviewing the previous literature on acupuncture treatment of sciatica, our assistance team produced initial clinical issues by convening video conferences and group discussions. And then, two methodological experts (WC and L-Q W) and

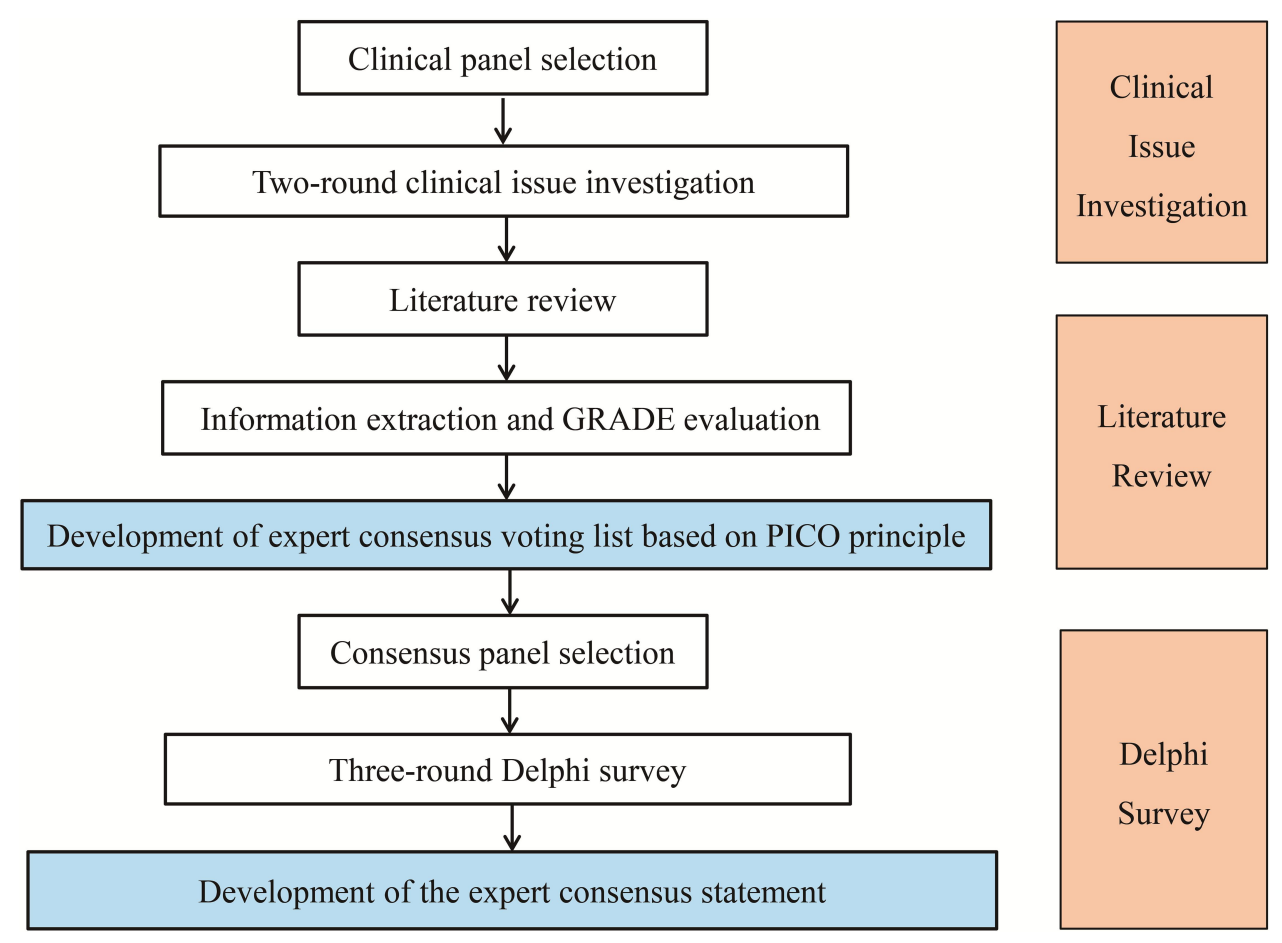

Figure I The development process of expert consensus. 
three acupuncture experts (C-Z L, HH, and YL) were invited to guide the initial clinical issues by email, and the settings of the relevant issues were revised repeatedly by acupuncture Ph.D. (NZ) according to the feedback of experts. After several revisions, a semi-open clinical questionnaire was generated with clinical problems that were classified according to PICO principles (patients, intervention, control, and outcomes).

\section{Clinical Panel Selection}

Before the clinical issue investigation for acupuncture treatment of sciatica, a group of acupuncture clinicians from the China Association of Acupuncture and orthopedic specialists were invited to participate in providing a series of questions. Meanwhile, they were also offered the opportunity to revise existing research questions or propose new questions for optimization of the later questionnaire, if they chose to.

\section{Two-Round Clinical Issue Investigation}

Two rounds of clinical issue investigation stage are from February 4, 2020 to March 24, 2020. The whole clinical research stage was divided into two rounds, which were sent to the acupuncture clinicians through the online software (www.wjx.cn - a professional online questionnaire survey, evaluation, and voting platform). After analyzing and summarizing the results of the first round, the contents of the second round of questionnaires are formulated based on the feedback of the controversial results in the first round. Finally, the results of the two rounds are analyzed and summarized to draft an initial expert consensus voting list (Table 1).

\section{Literature Review Retrieval}

Before voting on the consensus statements, a comprehensive literature search for systematic reviews based on PubMed, the Cochrane Library, and the Embase from January 2000 to March 2020 was conducted to seek documentary evidence support for the consensus voting lists using the specific search items (acupuncture OR acupuncture therapy OR body acupuncture OR manual acupuncture OR electroacupuncture OR electro-acupuncture OR warm needling) AND (sciatica OR sciatic neuralgia OR sciatica, bilateral OR neuralgia, sciatic OR bilateral sciatica) AND (systematic review OR meta-analysis)). Two reviewers independently extracted data and appraised the quality of the systematic reviews by applying AMSTAR- $2^{18}$ before grading the level of evidence by using the Grading of Recommendations, Assessment, Development, and Evaluation (GRADE). ${ }^{19}$
Table I The Initial Expert Consensus Voting Lists

\begin{tabular}{|c|c|}
\hline No & Statements \\
\hline I & $\begin{array}{l}\text { For the mild, moderate and severe sciatica caused by lumbar } \\
\text { disc herniation, acupuncture is recommended to improve the } \\
\text { clinical symptoms (lumbocrural pain, limb numbness, activity } \\
\text { disorder, sensory abnormality, muscle strength reduction, etc.) }\end{array}$ \\
\hline 2 & $\begin{array}{l}\text { For the mild, moderate and severe sciatica caused by lumbar } \\
\text { disc herniation, acupuncture is recommended to improve the } \\
\text { quality of life and mental state. }\end{array}$ \\
\hline 3 & $\begin{array}{l}\text { The treatment of sciatica for acupuncture, it is suggested to } \\
\text { meridians differentiation from the bladder meridian of zu tai } \\
\text { yang and gallbladder meridian of zu shao yang. }\end{array}$ \\
\hline 4 & $\begin{array}{l}\text { The treatment of sciatica for acupuncture should be based on } \\
\text { syndrome differentiation: syndrome of cold-dampness blocking } \\
\text { collaterals, syndrome of qi stagnation and blood stasis and } \\
\text { syndrome of deficiency of liver and kidney. }\end{array}$ \\
\hline 5 & $\begin{array}{l}\text { It is suggested to pay attention to the application of points } \\
\text { along meridians. The suggested acupoints are yao jia ji (EX-B2), } \\
\text { wei zhong (BL40), huan tiao (GB30), Yanglingquan (GB34), Zhi } \\
\text { bian (BL54), Cheng shan (BL57). }\end{array}$ \\
\hline 6 & $\begin{array}{l}\text { It is suggested to pay attention to the application of Ashi point. } \\
\text { The suggested number of Ashi is } 2-3 \text {. }\end{array}$ \\
\hline 7 & $\begin{array}{l}\text { The recommended reinforcing and reducing manipulations of } \\
\text { acupuncture therapy is: even reinforcing-reducing method. }\end{array}$ \\
\hline 8 & $\begin{array}{l}\text { The number of acupuncture points is recommended to select } \\
6-9 \text { acupoints. }\end{array}$ \\
\hline 9 & $\begin{array}{l}\text { The acupuncture stimulation is recommended to last for } \\
30 \mathrm{~min} \text {. }\end{array}$ \\
\hline 10 & $\begin{array}{l}\text { The frequency of acupuncture is recommended to last for } 3 \\
\text { sessions per week. }\end{array}$ \\
\hline II & $\begin{array}{l}\text { The period of acupuncture treatment is recommended to last } \\
\text { for } 4 \text { weeks. }\end{array}$ \\
\hline 12 & $\begin{array}{l}\text { The selection of acupoint is an important factor affecting the } \\
\text { therapeutic effect of acupuncture. }\end{array}$ \\
\hline 13 & $\begin{array}{l}\text { The depth of acupuncture is an important factor affecting the } \\
\text { therapeutic effect of acupuncture. }\end{array}$ \\
\hline 14 & $\begin{array}{l}\text { De } Q \mathrm{i} \text { is an important factor affecting the therapeutic effect of } \\
\text { acupuncture. }\end{array}$ \\
\hline 15 & $\begin{array}{l}\text { It is recommended to combine other traditional Chinese } \\
\text { medicine therapy to improve the clinical effect, such as } \\
\text { moxibustion, bleeding therapy, etc. }\end{array}$ \\
\hline 16 & $\begin{array}{l}\text { It is recommended that doctors should have the qualification } \\
\text { certificate of Chinese medicine practitioners and have more } \\
\text { than } 3 \text { years of experience. }\end{array}$ \\
\hline
\end{tabular}

(Continued) 
Table I (Continued).

\begin{tabular}{|l|l|}
\hline No & Statements \\
\hline 17 & $\begin{array}{l}\text { For the treatment of sciatica, the acupuncture has less adverse } \\
\text { reactions. Possible adverse reactions include: subcutaneous } \\
\text { hematoma, abnormal feeling after acupuncture (such as pain, } \\
\text { numbness, etc.), stagnation of acupuncture, bleeding, etc. }\end{array}$ \\
\hline
\end{tabular}

The quality of systematic reviews was divided into were "high-level evidence", "intermediate evidence", "low-level evidence" and "very low evidence". After the completion of cross-checking, if there is disagreement, the final result can be discussed through discussion or the participation of the third researcher. Finally, the consensus items were proposed according to the evidence and GRADE rating for the panel to make decisions.

\section{Delphi Consensus Survey Stage Consensus Panel Selection}

The experts who participated in the consensus stage are a group of scholars with the titles of deputy senior and above, who have considerable influence in the acupuncture and sciatica field of China with more than 10 years of experience in acupuncture. Before this survey, the consensus experts were contacted via an invitation email or WeChat (a cross-platform communication tool) to ensure that they are willing to participate in this survey. Then the expert opinions and voting results were collected through online software (www.wjx.cn).

\section{Delphi Survey}

An improved Delphi technology, a structured and verified identification consensus system, is used to collect expert questionnaire results and extract relevant information. ${ }^{20}$ The three-round Delphi survey based on consensus voting lists with each item accompanied by the results of investigation and literature evidence through online software was sent to consensus experts between April 5 and 25, 2020. To ensure the integrity of information collection, the experts were required to fill in their real names in each round, and make choices of "agree", "neutral", or "disagree" for each item based on their clinical experience and current clinical evidences, and explain the reasons for their choice. We defined the standard for reaching a consensus statement as the threshold of agreement expert consent should $>80 \%{ }^{21}$ In the first round, we provided a generic consensus voting list. After the first round of voting, if the proportion of items "agreeing" meets the consensus criteria, there is no need to continue the Delphi survey. Based on the statistical analysis results for the previous round of the Delphi survey, the items that have not reached consensus $(\leq 80 \%$ agreement) still need to proceed to the next round of consensus voting. The final consensus statements were reached by three rounds of Delphi investigation.

\section{Formulation of the Final Expert Consensus Statements}

The final generation of expert consensus is based on the results of three rounds of consensus survey, and our assistant team was responsible for drafting the first draft. After that, it was sent to the members of the expert group who participated in the voting by email. After the final review, discussion, and revision, the final expert consensus statements were formed.

\section{Results}

\section{The Clinical Issue Investigation}

Our assistance team raised a series of clinically related questions about acupuncture treatment of sciatica based on brainstorming and meeting discussions. After the advice and guidance of relevant experts, a clinical questionnaire was finally generated based on the PICO principle, mainly including the following aspects: 1) Intervention population 2) Acupuncture measures 3) Outcome indicators 4) Adverse reactions (shown in Supplementary Materials, Table S1).

Of the 80 potential attendees, 65 acupuncture clinicians, 41 females and 24 males (response rate: $81.25 \%$, $65 / 80$ ) confirmed their participation in the first round (20 from East China, 3 from South China, 20 from North China, 4 from Central China, 12 from Northeast China, 4 from Southwest China, and 2 from Northwest China). The number of senior title people was $23(35.83 \%)$, and the number of people who had worked for more than 10 years was $44(60.69 \%)$. The response rate for the second round of questionnaires was $76.92 \%$ (50/65). The proportions of women, geographical distribution, professional titles, etc., are similar to the first round. The detailed characteristics of acupuncture clinicians are shown in Table 2.

\section{Literature Review Retrieval}

Only two systematic reviews conformed to our search strategy and contained extractable data of interesting results, which were eventually included in our evidence report (The prism flowchart was shown in Supplementary Materials, Figure 1S). The results showed that the quality of the evidences was very low according to the GRADE 
Table 2 Baseline Characteristics of the Panel

\begin{tabular}{|c|c|c|c|}
\hline \multirow[t]{2}{*}{ Variables } & \multicolumn{3}{|c|}{ Voting Panel } \\
\hline & Round I & Round 2 & Consensus Stage \\
\hline Women, n (\%) & $4 \mathrm{I}(63.08 \%)$ & $32(64.00 \%)$ & $12(40.00 \%)$ \\
\hline \multicolumn{4}{|c|}{ Geographical distribution, n (\%) } \\
\hline East China & $20(30.77 \%)$ & $17(34.00 \%)$ & $9(30.00 \%)$ \\
\hline South China & $3(4.62 \%)$ & $3(6.00 \%)$ & I (3.33\%) \\
\hline North China & $20(30.77 \%)$ & $15(30.00 \%)$ & $10(33.33 \%)$ \\
\hline Central China & $4(6.15 \%)$ & $4(8.00 \%)$ & $4(13.33 \%)$ \\
\hline Northeast China & $12(18.46 \%)$ & $8(16.00 \%)$ & $3(10.00 \%)$ \\
\hline Southwest china & $4(6.15 \%)$ & I (2.00\%) & $3(10.00 \%)$ \\
\hline Northwest China & $2(3.08 \%)$ & $2(4.00 \%)$ & $0(0.00 \%)$ \\
\hline \multicolumn{4}{|c|}{ Highest qualification, n (\%) } \\
\hline Bachelor's degree & 5 (7.69\%) & $6(12.00 \%)$ & $2(6.67 \%)$ \\
\hline Master's degree & $18(27.69 \%)$ & $15(30.00 \%)$ & 7 (23.33\%) \\
\hline $\mathrm{PhD}$ & $42(64.62 \%)$ & $29(58.00 \%)$ & $21(70.00 \%)$ \\
\hline \multicolumn{4}{|c|}{ Professional title, n (\%) } \\
\hline Intermediate title & $17(26.15 \%)$ & $12(24.00 \%)$ & $0(0.00 \%)$ \\
\hline Deputy senior title & $25(38.46 \%)$ & $20(40.00 \%)$ & $2(6.67 \%)$ \\
\hline Senior title & $23(35.38 \%)$ & $18(36.00 \%)$ & $28(93.33 \%)$ \\
\hline \multicolumn{4}{|c|}{ Acupuncture practical experience (years), M (SD) } \\
\hline $3-10$ years & $21(32.31 \%)$ & $15(30.00 \%)$ & $0(0.00 \%)$ \\
\hline $\mathrm{II}-20$ years & $31(47.69 \%)$ & $23(46.00 \%)$ & 9 (30.00\%) \\
\hline $21-30$ years & $11(16.92 \%)$ & $10(20.00 \%)$ & $9(30.00 \%)$ \\
\hline$>30$ years & $2(3.08 \%)$ & $2(4.00 \%)$ & $12(40.00 \%)$ \\
\hline
\end{tabular}

(Reliability of system review results were shown in Supplementary Materials, Table S2). Finally, by analyzing and discussing the results of two rounds of the clinical questionnaire survey, an initial expert consensus voting list with 17 items was generated. The results of the systematic review were attached to the corresponding items of the initial expert consensus voting list as evidence. Items without evidence were accompanied by the results of two rounds of the clinical questionnaire stage.

\section{Delphi Consensus Survey Stage}

During the three rounds of the Delphi consensus survey stage, 30 experts were invited to participate in the survey, of which the ratio of women was $12(40.00 \%)$, the number of senior title people was $28(93.33 \%)$, and the number of people who had worked for more than 10 years was 30 $(100 \%)$. The detailed characteristics of the experts are shown in Table 2.

Of the 30 experts, $30(100 \%)$ completed the first, and third rounds, 29 (96.67\%) completed thesecond round while one of the experts did not fill it out for personal reasons. In round 1, expert consensus ( $>80 \%$ agreement) was reached on 13 of the 17 items included in the online questionnaire. Four items continued to be discussed in round 2 due to the lack of agreement. After thesecond round of expert consensus voting, 2 items reached aconsensus of more than $80 \%$. The other 2 items ( $\leq 80 \%$ agreement) were reserved for evaluation and discussion in round 3 . One item was reached on the consensus. Figures 2-4 showed the level of agreement for each of the items in the online questionnaire. Eventually, among the final consensus items, only 1 item was excluded from the 17 items.

\section{Formulation of the Final Expert Consensus Statements}

The final expert consensus statement includes 16 items, which can be roughly divided into the following: 1) The principle of acupuncture treatment for sciatica, combining syndrome differentiation with meridian differentiation. 2) The "dose" of acupuncture treatment for sciatica, mainly including acupoint selection, acupuncture frequency and duration time, etc. 3) The clinical effects of acupuncture on sciatica include not only the improvement of clinical 


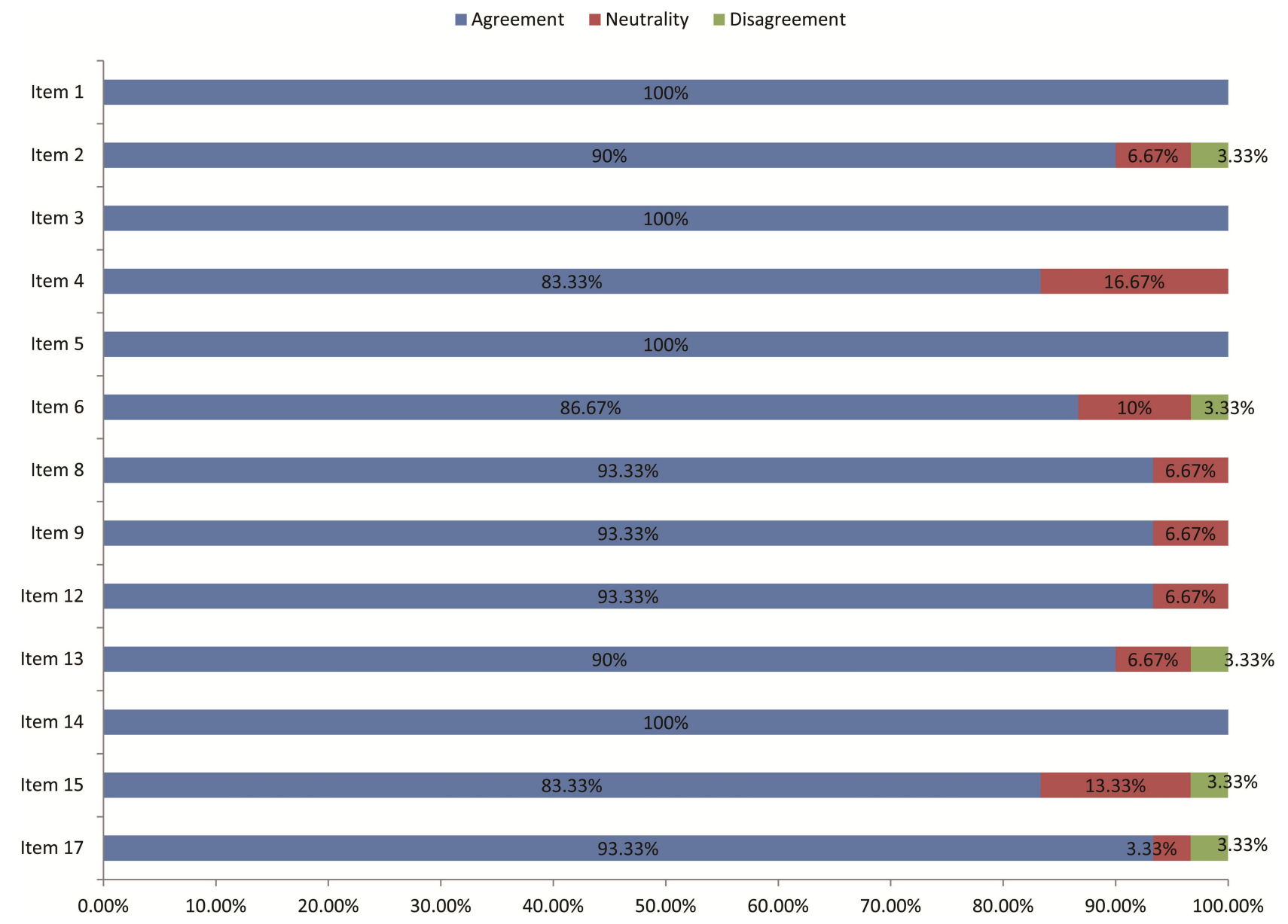

Figure 2 Results of first round of the Delphi consensus survey

symptoms but also the changes in patients' quality of life and mental state. 4) Adverse effects of acupuncture, such as bleeding, subcutaneous hematoma, etc. 5) others, such as the qualifications of acupuncturists.

\section{Discussion}

The purpose of this study is to establish a consensus based on the consensus of experts to determine the common clinical influencing factors and the best acupuncture parameters for acupuncture treatment of sciatica. The results showed that acupuncture therapy may have a satisfactory effect in improving sciatica. Nearly $90 \%$ of experts believe that the current acupuncture treatment can improve the pain and function of sciatica regardless of the severity of the disease that is to minimize the impact of sciatica on patients' life treatment. Although some terms specific to "traditional Chinese medicine" are included in the consensus items, the consensus result still has certain practicability and promotion significance.
According to the final consensus, acupuncture is recommended to improve the clinical symptoms for mild, moderate, and severe sciatica caused by the lumbar disc herniation. This consensus is similar to some previous research results. A single-center, parallel, randomized controlled trial involving 46 patients with sciatica pain for at least 12 weeks found that acupuncture was safer than sham acupuncture in treating chronic sciatica and had a curative effect in improving short-term pain. ${ }^{22}$ Two systematic reviews (one included 12 RCTs, 1842 patients; another included 11 RCTs, 932 patients) showed that acupuncture might be more effective in treating sciatica than drug therapy in relieving leg pain. ${ }^{23,24}$

Pain is the most common symptom of sciatica. As an emotional experience, it was defined as unpleasant feelings and emotional experiences related to actual or potential damage, or described by such damage. ${ }^{25}$ A recommendation from the Initiative on Methods, Measurement, and Pain Assessment in Clinical Trials (IMMPACT) that six-core outcome domains for the design of chronic pain clinical trials 


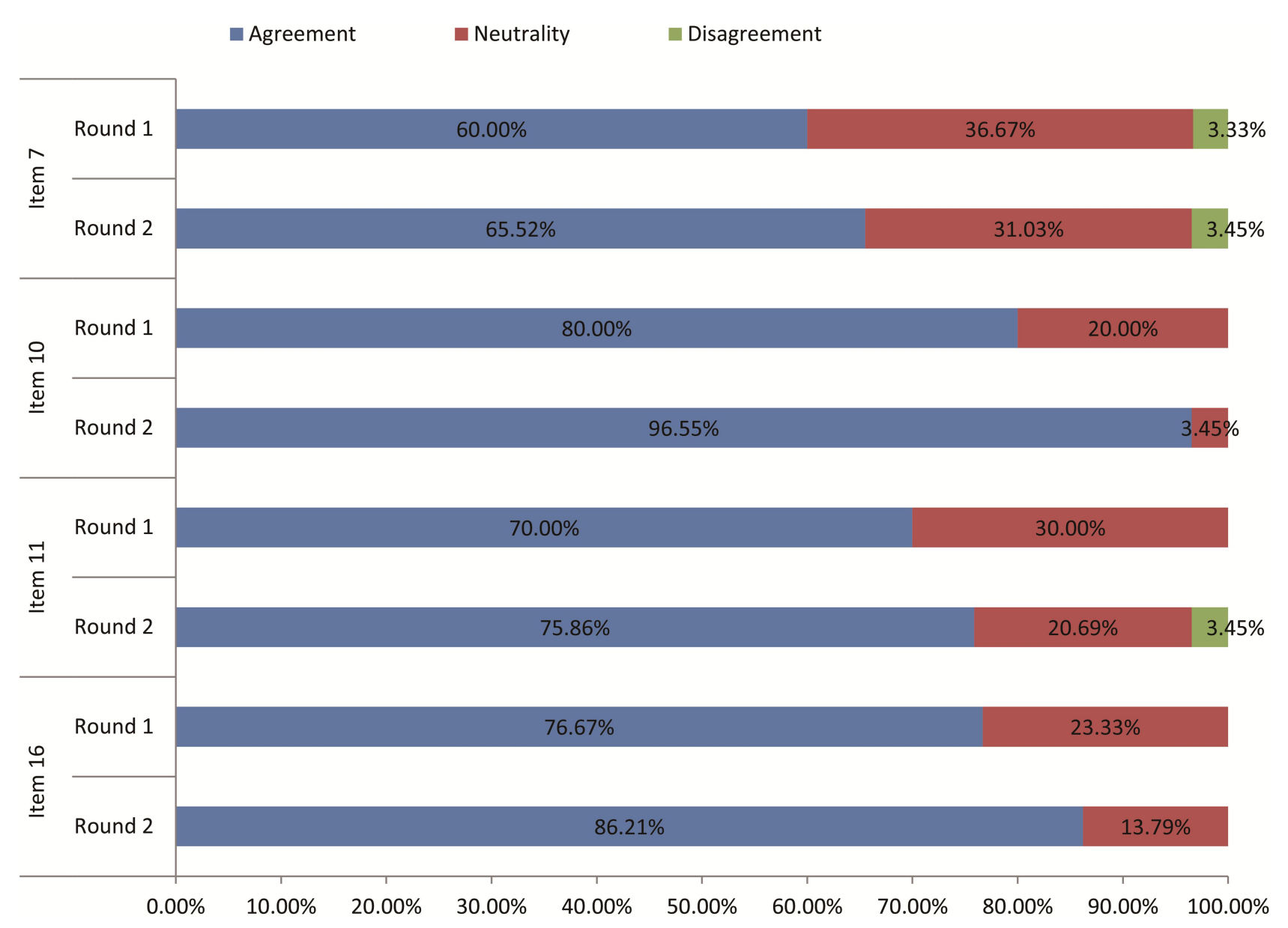

Figure 3 Results of two rounds of the Delphi consensus survey.

should be considered, including emotional functioning. ${ }^{26}$ Patients with chronic pain are prone to mental disorders and aggravate the psychological burden. ${ }^{27}$ In our consensus content, we also reached a consensus on the mental state. In the future clinical research for acupuncture treatment of sciatica, we must also pay attention to mental functioning.

Unlike conventional medical treatment, whether acupuncture can be effective for sciatica may be related to "dose". Based on traditional Chinese acupuncture theory, the number of acupuncture points, the selection of acupoints, the frequency of treatment, and the time of needle retention are the key factors to achieve the best treatment effect. ${ }^{28}$ A randomized controlled pilot study of the doseeffect of acupuncture on sciatica found that the number of acupuncture points (ie, dose) may play a key role in pain relief. The "low-dose" manual acupuncture (MAL) treatment group, which involved six acupoints, was as effective as the "high-dose" manual acupuncture (MAH) treatment group, which involved 18 acupoints in treating sciatica. ${ }^{29}$ However, the possible dose-related response has not been fully explored in sciatica. Therefore, we formulate consensus items on the parameters required for the effect of acupuncture intervention and finally reach recommendations on certain items, including the frequency of acupuncture treatment, needle holding time, and treatment time.

According to Traditional Chinese Medicine (TCM) theory, syndrome differentiation including eight principles, Zang-fu, meridians, Qi and Blood, or Triple-Energizer, is one of the basic principles of individual diagnosis and treatment of acupuncture. ${ }^{30}$ In TCM, acupoints are a special part of the body's viscera meridians and blood transfusion. The meridian system is the channel through which qi and blood flow. In the practice of acupuncture, the corresponding acupoints should be selected according to the meridian differentiation. In this study, we reached the consensus that acupuncture treatment of sciatica should pay attention to syndrome differentiation and meridian syndrome differentiation, which reflects the uniqueness of TCM theory, and thus guarantees the efficacy of acupuncture treatment. 


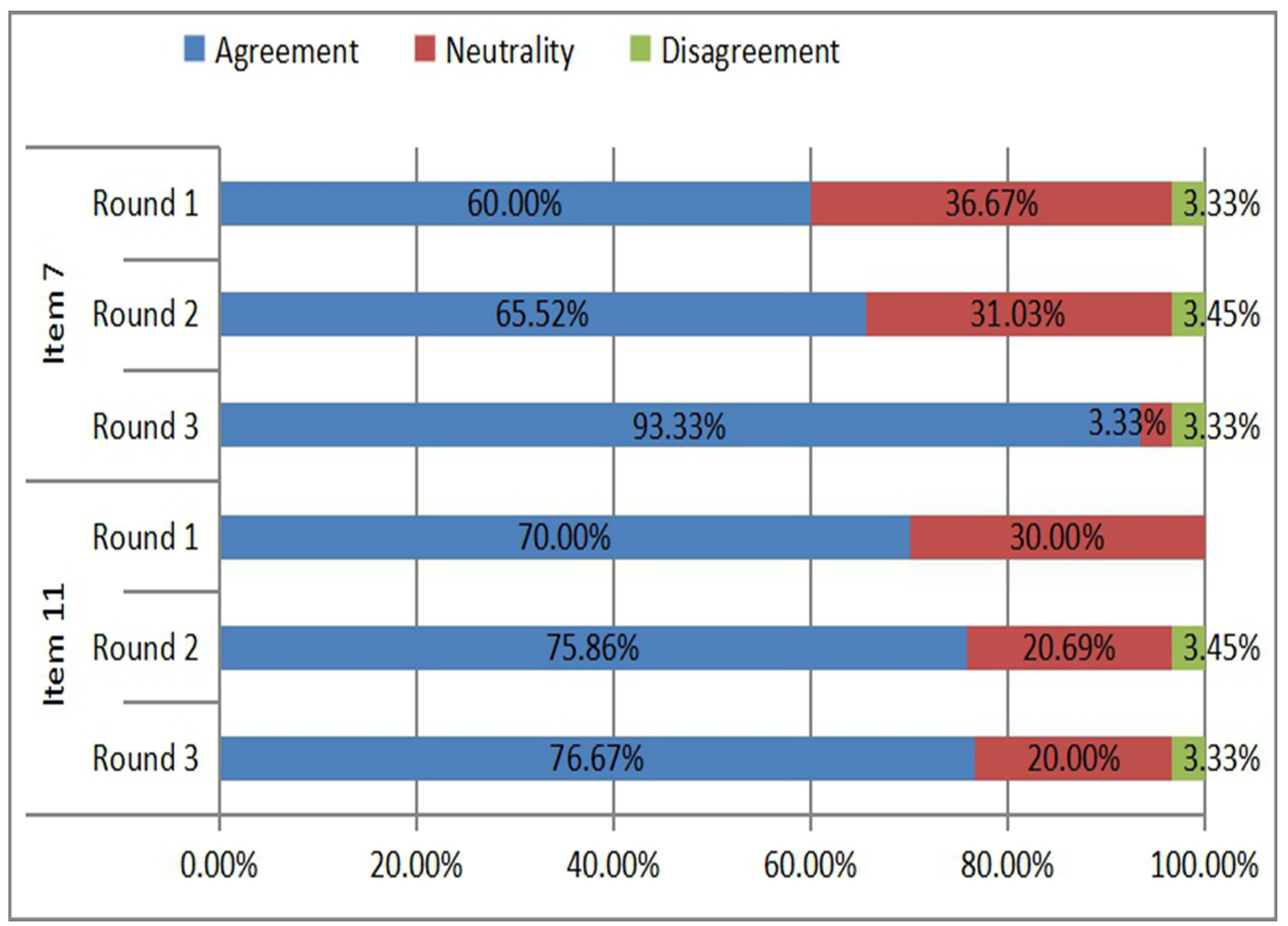

Figure 4 Results of three rounds of the Delphi consensus survey.

Although what is the optimal treatment method has always been the focus of acupuncture practitioners, ${ }^{31}$ however, due to the lack of sufficient evidence to promote the routine use of acupuncture treatment, there are many debates about acupuncture treatment of sciatica, partly because there is not enough high-quality evidence to fully explore possible dose-related responses. ${ }^{32,33}$ Therefore, after extensively soliciting the suggestions and opinions of multiple clinicians, our study formulated an expert consensus around the clinical-specific problems for acupuncture treatment of sciatica based on systematic review evidence.

\section{Strengths and Limitations}

This study has several strengths. Before starting the Delphi survey, the real need for acupuncture treatment of sciatica should be carefully evaluated, so the previous two rounds of clinical problem investigations were generated. Second, the evidence of consensus items was reviewed based on systematic reviews and clinical investigation results. Finally, our expert groups are different in composition.
The clinical problem investigation stage is that clinicians from different regions of China can provide a wider range of clinical opinions and suggestions. Our 30 experts of the consensus stage are the professors with a well known in Chinese acupuncture.

This study also has certain limitations. Despite the careful selection of panel members and following the Delphi methodology rigorously, the results of this consensus may not cover the opinion of the entire acupuncture community but have aimed at the best possible synthesis at the time. Regarding the selection of expert sampling, due to the large number of items involving acupuncture operations and parameters, only experts in acupuncture were included, and experts who are professionals in sciatica but do not practice acupuncture were not included. A multidisciplinary expert committee is necessary to discuss the application of acupuncture. Since our survey is currently only a preliminary version, we will consider the necessity of communicating with the multidisciplinary expert committee in the next consensus 
survey. The systematic reviews supported by evidence cannot provide strong support for our consensus items due to its low quality, which has also contributed to our expert consensus to a certain extent. At the same time, since our expert groups were all from China, to a certain extent, the consensus's extrapolation to other countries was affected.

\section{Conclusion}

In conclusion, this consensus statement was in agreement that the treatment of acupuncture was an essential part of sciatica treatment and provided a useful tool for physicians to facilitate individualized treatment decisions regarding the management of sciatica. At the same time, due to the large individual differences of patients, our consensus is only a method to provide a new perspective for future clinical research on the effectiveness of acupuncture.

\section{Data Sharing Statement}

If you need the original data, please contact Cun-Zhi Liu, email: lcz_tg@126.com.

\section{Ethical Corrections}

Our study is a Delphi expert consensus survey but NOT a clinical trial. The participants are authoritative experts in the field. They just needed to put forward their opinions on the specific issues but NOT accepted any intervention. There is NOT any patient recruited in the study, either. In accordance with the legislation and institutional requirements, and the previous published articles, the ethical review is NOT required for this kind of study. The experts who confirmed their willing to participate in the Delphi survey had signed the written informed consents.

\section{Acknowledgments}

This study was supported by the National Key R\&D Program of China [grant number 2019YFC1712100].

\section{Author Contributions}

All authors made a significant contribution to the work reported, whether that is in the conception, study design, execution, acquisition of data, analysis and interpretation, or in all these areas; took part in drafting, revising or critically reviewing the article; gave final approval of the version to be published; have agreed on the journal to which the article has been submitted; and agree to be accountable for all aspects of the work.

\section{Disclosure}

The authors have declared no conflicts of interest.

\section{References}

1. Frymoyer JW. Back pain and sciatica. $N$ Engl $J$ Med. 1988;318:291-300.

2. Jensen Rikke K, Kongsted A, Kjaer P, et al. Diagnosis and treatment of sciatica. BMJ. 2019;367:16273. doi:10.1136/bmj.16273

3. Frymoyer JW. Lumbar disk disease: epidemiology. Instr Course Lect. 1992;41:217-223.

4. Koes BW, Van Tulder MW, Peul WC. Diagnosis and treatment of sciatica. BMJ. 2007;334(7607):1313-1317. doi:10.1136/bmj.39223.428495.BE

5. Valat JP, Genevay S, Marty M, et al. Sciatica. Best Pract Res Clin Rheumatol. 2010;24:241-252. doi:10.1016/j.berh.2009.11.00520227645

6. Porchet F, Wietlisbach V, Burnand B, et al. Relationship between severity of lumbar disc disease and disability scores in sciatica patients. Neurosurgery. 2002;50:1253-1259.

7. Ropper AH, Zafonte RD. Sciatica. New Engl J Med. 2015;372 (13):1225-1240. doi:10.1056/NEJMra1410151

8. Hartvigsen L, Hestbaek L, Lebouef-Yde C, et al. Leg pain location and neurological signs relate to outcomes in primary care patients with low back pain. BMC Musculoskelet Disord. 2017;18(1):133. doi:10.1186/s12891-017-1495-3

9. Vroomen PC, de Krom MC, Wilmink JT, et al. Lack of effectiveness of bed rest for sciatica. $N$ Engl J Med. 1999;340:418-423. doi:10.1056/NEJM199902113400602

10. Oliveira Crystian B, Maher Chris G, Pinto Rafael Z, et al. Clinical practice guidelines for the management of non-specific low back pain in primary care: an updated overview. Eur Spine $J$. 2018;27:2791-2803. doi:10.1007/s00586-018-5673-2

11. van der Gaag WH, Roelofs PDDM, Enthoven WTM, et al. Nonsteroidal anti-inflammatory drugs for acute low back pain. Cochrane Database Syst Rev. 2020;4. doi:10.1002/14651858.CD013581.

12. Brune K, Patrignani P. New insights into the use of currently available non-steroidal anti-inflammatory drugs. $J$ Pain Res. 2015;8:105-118. doi:10.2147/JPR.S75160.

13. Stochkendahl MJ, Kjaer P, Hartvigsen J, et al. National Clinical Guidelines for non-surgical treatment of patients with recent onset low back pain or lumbar radiculopathy. Eur Spine J. 2018;27:60-75. doi:10.1007/s00586-017-5099-2

14. Qaseem A, Wilt TJ, McLean RM, Forciea MA; Clinical Guidelines Committee of the American College of Physicians. Noninvasive Treatments for acute, subacute, and chronic low back pain: a clinical practice guideline from the American College of Physicians. Ann Intern Med. 2017;166(7):514-530. doi:10.7326/M16-2367

15. Peul WC, van Houwelingen HC, van den Hout WB, et al. Surgery versus prolonged conservative treatment for sciatica. $N$ Engl J Med. 2007;356(22):2245-2256. doi:10.1056/NEJMoa064039

16. de Campos TF. Low back pain and sciatica in over 16s: assessment and management NICE Guideline [NG59]. J Physiother. 2017;63 (2):120. doi:10.1016/j.jphys.2017.02.012.

17. China Association of Acupuncture-Moxibustion. Evidence-Based Guidelines of Clinical Practice with Acupuncture and Moxibustion: Sciatica. Beijing, China: China Press of Traditional Chinese Medicine; 2018.

18. Shea BJ, Reeves BC, Wells G, et al. AMSTAR 2: a critical appraisal tool for systematic reviews that include randomised or non-randomised studies of healthcare interventions, or both. BMJ. 2017;358:j4008. doi:10.1136/bmj.j4008.

19. Atkins D, Best D, Briss PA, et al. Grading quality of evidence and strength of recommendations. BMJ. 2004;328(7454):1490. doi:10.1136/bmj.328.7454.1490.

20. Hasson F, Keeney S, McKenna H. Research guidelines for the Delphi survey technique. J Adv Nurs. 2000;32(4):1008-1015. 
21. Hohmann E, Angelo R, Arciero R, et al. Degenerative meniscus lesions: an expert consensus statement using the modified delphi technique. Arthroscopy. 2020;36(2):501-512. doi:10.1016/j. arthro.2019.08.014.

22. Huang Z, Liu S, Zhou J, et al. Efficacy and safety of acupuncture for chronic discogenic sciatica, a randomized controlled Sham acupuncture trial. Pain Med. 2019;20(11):2303-2310. doi:10.1093/pm/ pnz167.

23. Ji M, Wang X, Chen M, et al. The efficacy of acupuncture for the treatment of sciatica: a systematic review and meta-analysis. Evid Based Complement Alternat Med. 2015;2015:192808. doi:10.1155/ 2015/192808.

24. Qin Z, Liu X, Wu J, et al. Effectiveness of acupuncture for treating sciatica: a systematic review and meta-analysis. Evid Based Complement Alternat Med. 2015;2015:425108. doi:10.1155/2015/ 425108.

25. Williams AC, Craig KD. Updating the definition of pain. Pain. 2016;157(11):2420-2423. doi:10.1097/j.pain.0000000000000613.

26. Turk DC, Dworkin RH, Allen RR, et al. Core outcome domains for chronic pain clinical trials: IMMPACT recommendations. Pain. 2003;106(3):337-345. doi:10.1016/j.pain.2003.08.001.

27. Demyttenaere K, Bruffaerts R, Lee S, et al. Mental disorders among persons with chronic back or neck pain: results from the World Mental Health Surveys. Pain. 2007;129(3):332-342. doi:10.1016/j. pain.2007.01.022.
28. Pomeranz B, Berman B. Scientific Basis of Acupuncture; Basics of Acupuncture. Springer; 2003:7-86.

29. Liu CH, Kung YY, Lin CL, et al. Therapeutic efficacy and the impact of the "Dose" effect of acupuncture to treat sciatica: a randomized controlled pilot study. J Pain Res. 2019;12:3511-3520. doi:10.2147/ JPR.S210672.

30. Cao H, Bourchier S, Liu J. Does syndrome differentiation matter? A meta-analysis of randomized controlled trials in cochrane reviews of acupuncture. Med Acupunct. 2012;24(2):68-76. doi:10.1089/ acu.2011.0846.

31. Hanfileti L. What licensed acupuncturists need to know about the training and qualifications of physicians providing medical acupuncture. Insights for Acupuncturists; 2013, https://www.insights-foracupuncturists.com/medical-acupuncture.html. Accessed December 8, 2020.

32. Zhao L, Chen J, Liu CZ, et al. A review of acupoint specificity research in china: status quo and prospects. Evid Based Complement Alternat Med. 2012;2012:543943. doi:10.1155/2012/ 543943.

33. Armour M, Smith CA. Treating primary dysmenorrhoea with acupuncture: a narrative review of the relationship between acupuncture 'dose' and menstrual pain outcomes. Acupunct Med. 2016;34 (6):416-424. doi:10.1136/acupmed-2016-011110.

\section{Publish your work in this journal}

The Journal of Pain Research is an international, peer reviewed, open access, online journal that welcomes laboratory and clinical findings in the fields of pain research and the prevention and management of pain. Original research, reviews, symposium reports, hypothesis formation and commentaries are all considered for publication. The manuscript management system is completely online and includes a very quick and fair peer-review system, which is all easy to use. Visit http:// www.dovepress.com/testimonials.php to read real quotes from published authors. 